\title{
On rigidity of locally symmetric spaces
}

\author{
Chris Peters \\ (Communicated by Peter Schneider)
}

\begin{abstract}
In this note I generalize the classical results of Calabi-Vesentini [3] (cp. also [2]) to certain noncompact locally symmetric domains, namely those that are quotients of a hermitian symmetric domain by a neat arithmetic subgroup of the group of its holomorphic automorphisms.
\end{abstract}

\section{INTRODUCTION}

A classical result due to Calabi and Vesentini [3] states that a compact locally symmetric space is rigid, provided all of its irreducible factors have dimension at least 2. This implies that such varieties (known to be algebraic) can be defined over a numberfield. This was first remarked by Shimura in [10]. For a modern variant of the proof see [7].

Faltings [5] remarked that one can show that the Kodaira-Spencer class for any "spread family" of the given variety is zero which suffices for rigidity. This is true without any restriction on the type of irreducible factors, and even for noncompact locally symmetric spaces. The proof uses first of all Mumford's theory of toroidal compactifications [1] of locally symmetric varieties together with the existence of "good" extensions of metric homogeneous vector bundles to these compactifications as shown in [6]. The second ingredient is a careful analysis of the extension of classical harmonic theory to a suitable $L^{2}$-version.

I show in this note that the same techniques can be used to extend the results of Calabi and Vesentini to the noncompact case. This is stated as Theorem 4.3.

Mumford's ideas are sketched in Section 1. In Section 2, I explain the basic $L^{2}$-techniques used by Faltings. This is done in some detail since the arguments in [5] are rather sketchy. 


\section{Poincaré Growth AND GOOD METRICS}

In this section I recall some concepts and results from [6]. Let $X$ be a smooth quasi-projective complex variety and let $\bar{X}$ be a "good" compactification: $\bar{X}$ is non-singular, projective and $\partial X:=\bar{X}-X$ a normal crossing divisor. Hence, locally at a point of the boundary, coordinates $\left(z_{1}, \ldots, z_{n}\right)$ can be chosen such that the boundary is given by the equation $z_{1} \cdots z_{r}=0$ and $\partial X$ can be covered by a collection of polydisks $\Delta^{n}$ on which $X$ cuts out $\left(\Delta^{*}\right)^{r} \times \Delta^{n-r}$. Let \|\|$_{P}$ be the Poincaré norm on such a product given on a coordinate disk $(\Delta, z)$ by the usual euclidian norm while on a punctured disk $\left(\Delta^{*}, z\right)$ it is given by $|z|^{2}(\log |z|)^{2}$.

Any smooth $p$ form, say $\eta$ on $X$, is said to have Poincaré growth near the boundary, if for all tangent vectors $\left\{t_{1}, \ldots, t_{p}\right\}$ at a point of $\Delta^{n} \cap X$, one has the estimate $\left|\eta\left(t_{1}, \ldots, t_{p}\right)\right|^{2} \leq$ Const. $\left\|t_{1}\right\|_{P} \cdots\left\|t_{p}\right\|_{P}$. This notion does not depend on choices. By [6, Prop. 1.1] such a form defines a current on $X$. Mumford calls a smooth form $\omega$ on $X$ a good form if $\omega$ as well as $d \omega$ have Poincaré growth near the boundary.

Let $(E, h)$ be a hermitian holomorphic vector bundle on $X$. Recall the following definition:

Definition 1.1. The Chern connection for $(E, h)$ is the unique metric connection $\nabla_{E}$ on $E$ whose $(0,1)$-part is the operator $\bar{\partial}: \mathcal{A}_{X}^{0}(E) \rightarrow \mathcal{A}_{X}^{0,1}(E)$ coming from the complex structure on $E$.

Assume that $E=\left.E^{\#}\right|_{X}$ where $E^{\#}$ is a holomorphic vector bundle on $\bar{X}$.

Definition 1.2. The metric $h$ is good relative to $E^{\#}$, if locally near the boundary for every frame of $E^{\#}$ the following holds:

(i) The matrix entries $h_{i j}$ of $h$, respectively $h_{i j}^{-1}$ of $h^{-1}$, with respect to the frame grow at most logarithmically: in local coordinates $z_{1}, \ldots, z_{n}$ as above, $\left|h_{i j}\right|,\left|h_{i j}^{-1}\right| \leq$ Const. $\cdot\left(\log \left|z_{1} \cdots z_{k}\right|\right)^{N}$ for some integer $N$.

(ii) The entries of the connection matrix $\omega_{h}=\partial h \cdot h^{-1}$ for the Chern connection are good forms (as defined above).

By [6, Prop. 1.3] there is at most one extension $E^{\#}$ of $E$ such that $h$ is good relative to that extension. Note also that the dual $E^{*}$ carries a natural metric and this metric is good relative $\left(E^{\#}\right)^{*}$.

If $h$ is a good metric on a vector bundle $E$ relative to an extension $E^{\#}$, then by definition any Chern form calculated from the Chern connection is good, and by [6, Thm. 1.4] the class it represents is the corresponding Chern class of $E^{\#}$.

\section{Relevant $L^{2}$-HARMonic theory}

Let me continue with the set-up of the previous section. So $(E, h)$ is a hermitian holomorphic vector bundle on $X$ such that $E$ is the restriction to $X$ of a holomorphic vector bundle $E^{\#}$ on $\bar{X}$ with the property that $h$ is good relative to $E^{\#}$. In addition, make the following, admittedly strong assumptions: 
Assumption 2.1. (a) $X$ carries a complete Kähler metric $h_{X}$ whose $(1,1)$ form has Poincaré growth near $\partial X$ (and hence its volume form has Poincaré growth).

(b) Smooth sections of the bundle $\mathcal{A}_{\bar{X}}\left(E^{\#}\right)$ of complex $k$-forms with values in $E^{\#}$ are bounded in the metric on the space of global sections that $h$ and $h_{X}$ induce as explained now.

On $A^{k}\left(E^{\#}\right)$, the space of global complex $k$-forms with values in $E^{\#}$, this goes as follows. On a fiber $\mathcal{A}_{X, x}^{k}(E)$ at $x \in X$ of the vector bundle $\mathcal{A}_{X}^{k}(E)$, one has a fiberwise metric induced by the metrics $h$ and $h_{X}$ :

$$
h_{x}(\alpha \otimes s, \beta \otimes t)=h_{X}(\alpha, \beta) h(s, t), \quad \alpha, \beta \in \mathcal{A}_{X, x}^{k}, s, t \in E_{x} .
$$

Assumption 2.1 (b) means that for any two sections $\omega_{i} \in A^{k}\left(E^{\#}\right), i=1,2$, the function $\left\{x \mapsto h_{x}\left(\omega_{1}, \omega_{2}\right)\right\}$ is bounded on $X$. Since by Assumption 2.1 (a) the volume form for $h_{X}$ has Poincaré growth near $\partial X$, it follows that the global inner product

$$
\left\langle\omega_{1}, \omega_{2}\right\rangle=\int_{X} h_{x}\left(\omega_{1}, \omega_{2}\right) \cdot \text { volume form with respect to } h_{X},
$$

$\omega_{1}, \omega_{2} \in A_{\bar{X}}^{k}\left(E^{\#}\right)$, exists; in other words, one has an inclusion

$$
A^{k}\left(E^{\#}\right) \hookrightarrow L^{2}\left(X, A^{k}(E)\right)=\{\text { square integrable } E \text {-valued } k \text { forms }\}
$$

and one can do harmonic theory for certain differential operators on these spaces. The particular operators here are those that are induced by the Chern connection $\nabla=\nabla_{E}$ (see Definition 1.1), namely

$$
\begin{aligned}
\nabla: \mathcal{A}_{X}^{k}(E) & \rightarrow \mathcal{A}_{X}^{k+1}(E), \quad \nabla^{0,1}=\bar{\partial}, \\
\alpha \otimes s & \mapsto d \alpha \otimes s+(-1)^{k} \alpha \otimes \nabla s .
\end{aligned}
$$

The operator $\bar{\partial}$ extends in the distributional sense to an operator

$$
\bar{\partial}: L^{2}\left(X, A^{0, q}(E)\right) \rightarrow L^{2}\left(X, A^{0, q+1}(E)\right),
$$

and since the metric on $X$ is complete and $\bar{\partial}^{2}=0$, one can apply a result of von Neumann (cp. $[4, \S 12])$ which says that there is a formal adjoint operator $\bar{\partial}^{*}: L^{2}\left(X, A^{0, q+1}(E)\right) \rightarrow L^{2}\left(X, A^{0, q}(E)\right)$ in the sense of distributions. Moreover, the formal adjoint of $\bar{\partial}^{*}$ exists and equals $\bar{\partial}$. These adjoints, viewed as operators on the bundles $\mathcal{A}_{X}^{0, *}(E)$, coincide with the classical ones:

Lemma 2.2. Let $*_{E}: \mathcal{A}_{X}^{p, q}(E) \rightarrow \mathcal{A}_{X}^{n-q, n-p}(E)$ be the fiberwise defined operator induced by the Hodge star-operator.

(i) The formal adjoint $\bar{\partial}^{*}$ is induced by

$$
-*_{E} \nabla^{1,0} *_{E}: \mathcal{A}_{X}^{0, q+1}(E) \rightarrow \mathcal{A}_{X}^{0, q}(E) .
$$

(ii) The formal adjoint of $\nabla^{1,0}$ equals $\left(\nabla^{1,0}\right)^{*}=-*_{E} \bar{\partial} *_{E}$. 
Proof. Since $\bar{\partial}=-\left(*_{E} \nabla^{1,0} *_{E}\right)^{*}=-*_{E}\left(\nabla^{1,0}\right)^{*} *_{E}$, the second assertion follows from the first. The meaning of the first assertion is that for $\omega_{1} \in A^{0, q}\left(E^{\#}\right)$ and $\omega_{2} \in A^{0, q+1}\left(E^{\#}\right)$ one has

$$
\left\langle\bar{\partial} \omega_{1}, \omega_{2}\right\rangle=-\left\langle\omega_{1},\left(*_{E} \nabla^{1,0} *_{E}\right) \omega_{2}\right\rangle .
$$

To show this, let me go through the classical calculation. First, using the metric contraction

$$
\begin{aligned}
h_{E}: A^{k}(E) \otimes A^{\ell}(E) & \rightarrow A^{k+\ell}, \\
(\alpha \otimes s, \beta \otimes t) & \mapsto h_{E}(s, t) \alpha \wedge \bar{\beta},
\end{aligned}
$$

one observes the fundamental equation

(2) $h_{E}\left(\varphi_{1}, *_{E} \varphi_{2}\right)=h_{x}\left(\varphi_{1}, \varphi_{2}\right) \cdot$ volume form $d V, \quad x \in X, \varphi_{1}, \varphi_{2} \in A^{k}(E)$.

Next, the Chern connection being metric implies that for the forms restricted to $X$ (denoted by the same symbols) one has

$$
h_{E}\left(\nabla \omega_{1}, *_{E} \omega_{2}\right)+(-1)^{k} h_{E}\left(\omega_{1}, \nabla\left(*_{E} \omega_{2}\right)\right)=d h_{E}\left(\omega_{1}, *_{E} \omega_{2}\right),
$$

and hence, using (2) and the relation $*_{E} \cdot *_{E}=(-1)^{k}$, one finds

$$
\bar{\partial} h_{E}\left(\omega_{1}, *_{E} \omega_{2}\right)=\left[h_{x}\left(\bar{\partial} \omega_{1}, \omega_{2}\right)+h_{x}\left(\omega_{1},\left(*_{E} \nabla^{1,0} *_{E}\right) \omega_{2}\right)\right] \cdot d V .
$$

I claim that $\bar{\partial} h_{E}\left(\omega_{1}, *_{E} \omega_{2}\right)$ is bounded near $\partial X$ and that it integrates over $X$ to zero. Assume this for a moment. Since the first term on the right is bounded, the other is too. Hence after integration one obtains

$$
0=\left\langle\bar{\partial} \omega_{1}, \omega_{2}\right\rangle+\left\langle\omega_{1},\left(*_{E} \nabla^{1,0} *_{E}\right) \omega_{2}\right\rangle
$$

and the result follows.

It remains to show the assertion about $\bar{\partial} h_{E}\left(\omega_{1}, *_{E} \omega_{2}\right)$. Let $U_{\delta}$ be a tubular neighborhood of $\partial X$ with radius $\delta$. By Stokes' theorem,

$$
\int_{X} \bar{\partial} h_{E}\left(\omega_{1}, *_{E} \omega_{2}\right)=\lim _{\delta \rightarrow 0} \int_{\partial U_{\delta}} h_{E}\left(\omega_{1}, *_{E} \omega_{2}\right)=0 .
$$

The last equality follows since by (2) the integrand has Poincaré growth near the boundary and hence the integral tends to zero (compare the proof of [6, Prop. 1.2]).

The Laplacian $\Delta_{E}:=\bar{\partial} \bar{\partial}^{*}+\bar{\partial}^{*} \bar{\partial}$ preserves $L^{2}\left(A^{0, q}(X)\right)$ and the forms $\omega$ with $\Delta_{E} \omega=0$ are by definition the harmonic forms. Reasoning as in the classical situation (cp. $[4, \S 12])$ one shows:

Corollary 2.3. (i) For all $\omega \in A_{\bar{X}}^{0, q}\left(E^{\#}\right)$ one has

$$
\left\langle\Delta_{E} \omega, \omega\right\rangle=\langle\bar{\partial} \omega, \bar{\partial} \omega\rangle+\left\langle\bar{\partial}^{*} \omega, \bar{\partial}^{*} \omega\right\rangle .
$$

Hence, in the distributional sense, one has

$$
\Delta_{E} \omega=0 \Longleftrightarrow \bar{\partial} \omega=0=\bar{\partial}^{*} \omega .
$$


(ii) There is an orthogonal decomposition

$$
L^{2}\left(X, \mathcal{A}_{X}^{0, q}(E)\right)=\left[\bar{\partial} A_{X}^{0, q-1}(E)\right]^{\mathrm{cl}} \oplus\left[\bar{\partial}^{*} A_{X}^{0, q+1}(E)\right]^{\mathrm{cl}} \oplus \mathrm{H}_{(2)}^{0, q}(E),
$$

where the symbol ${ }^{\mathrm{cl}}$ stands for "topological closure" and the symbol $\mathrm{H}_{(2)}$ stands for the harmonic $L^{2}$-forms, i.e. $L^{2}$-forms $\omega$ with $\Delta_{E} \omega=0$ in the sense of distributions.

To apply this, recall that by Dolbeault's theorem the cohomology group $H^{k}\left(\bar{X}, E^{\#}\right)$ can be calculated as the $k$-th cohomology of the complex $\mathcal{A}_{\bar{X}}^{0, *}\left(E^{\#}\right)$.

Proposition 2.4 ([5, Lem. 2]). Assume that $\bar{E}$ is a holomorphic vector bundle on $\bar{X}$ and that $\left(E=\left.E^{\#}\right|_{X}, h\right)$ is a hermitian bundle on $X$ such that $h$ is good relative $E^{\#}$. If Assumption 2.1 holds, then there is a natural injective homomorphism

$$
j_{L^{2}}^{*}: H^{k}\left(\bar{X}, E^{\#}\right)=H^{k}\left(\mathcal{A}_{\bar{X}}^{0, *}\left(E^{\#}\right)\right) \rightarrow \mathrm{H}_{(2)}^{0, k}(X, E),
$$

with target the space of E-valued harmonic square integrable $(0, k)$-forms.

Proof. The map $j_{L^{2}}^{*}$ is induced from the orthogonal projection to $\mathrm{H}_{L^{2}}^{k}(E)$. The procedure is as follows. Pick $\alpha \in \mathcal{A}_{\bar{X}}^{0, k}\left(E^{\#}\right)$ for which $\bar{\partial} \alpha=0$ representing a given cohomology class $[\alpha] \in H^{k}\left(\bar{X}, E^{\#}\right)$. By Assumption $2.1, \beta=\alpha \mid X$ is an $E$-valued $L^{2}$-form whose orthogonal projection to the harmonic forms is $j_{L^{2}}^{*} \alpha$. One needs to verify independence of choices: since $\bar{\partial} \alpha=0$, one has $\bar{\partial} \beta=0$ in the sense of currents and so, another representative for $\alpha$ leads to a form which differs from $\beta$ by a current of the form $\bar{\partial} \gamma$. Hence the harmonic projection is independent of choices.

To see that it is injective, suppose that the harmonic part of $\beta$ vanishes. By (1) one has $\left\langle\beta, \bar{\partial}^{*} \varphi\right\rangle=\langle\bar{\partial} \beta, \varphi\rangle=0$ and hence $\beta$ belongs to the first summand of (3) so that

$$
\beta=\lim _{j \rightarrow \infty} \bar{\partial} \gamma_{j}, \quad \gamma_{j} \in \mathcal{A}_{\bar{X}}^{0, k-1}\left(E^{\#}\right)
$$

To test that this gives the zero class in $H^{k}\left(\bar{X}, E^{\#}\right)$, one uses the Serre duality pairing:

$$
H^{k}\left(\bar{X}, E^{\#}\right) \otimes H^{n-k}\left(\bar{X}, \Omega_{\bar{X}}^{n} \otimes\left(E^{*}\right)^{\#}\right) \rightarrow H^{n, n}(\bar{X})=\mathbf{C}
$$

as induced by the pairing

$$
\mathcal{A}_{\bar{X}}^{0, k}\left(E^{\#}\right) \otimes \mathcal{A}_{\bar{X}}^{0, n-k}\left(\Omega \frac{n}{X} \otimes\left(E^{*}\right)^{\#}\right) \rightarrow \mathcal{A}_{\bar{X}}^{n, n} .
$$

To this end, consider a closed $\beta^{\prime} \in \mathcal{A}_{\bar{X}}^{0, n-k}\left(\Omega_{\frac{n}{X}} \otimes\left(E^{*}\right)^{\#}\right)$. I claim that near $\partial X$ it is bounded in norm.

To see this, one uses that $\Omega \frac{n}{X}(\log \partial X) \otimes\left(E^{*}\right)^{\#}$ is the unique extension of the bundle $\Omega_{X}^{n} \otimes E^{*}$ on $X$ for which $h=h_{X} \otimes h_{E^{*}}$ is good. That this is the case will be shown later (see Example $4.2(\mathrm{i})$ ). Let $f$ be a local equation for $\partial X$. Then a local expression for $\beta^{\prime}$ is of the form $f \cdot \gamma \cdot s$ with $\gamma$ a bounded form of type $(0, n-k)$ and $s$ a local section of $\Omega \frac{n}{X}(\log \partial X) \otimes\left(E^{*}\right)^{\#}$. Since $h(f \cdot s, f \cdot s)=$ $|f|^{2} h(s, s)$ and $h(s, s)$ has logarithmic growth near $\partial X$, it follows that $h\left(\beta^{\prime}, \beta^{\prime}\right)$ 
tends to zero when one approaches $\partial X$. Hence $\beta^{\prime} \in L^{2}\left(A_{X}^{0, n-k}\left(\Omega_{X}^{n} \otimes E^{*}\right)\right)$. The Serre pairing therefore is given by

$$
\left(\beta, \beta^{\prime}\right):=\lim _{j \rightarrow \infty} \int_{X} \bar{\partial} \gamma_{j} \wedge \beta^{\prime}=\lim _{j \rightarrow \infty} \lim _{\delta \rightarrow 0} \int_{\partial U_{\delta}} \gamma_{j} \wedge \beta^{\prime},
$$

where $U_{\delta}$ is a tubular neighborhood of $\partial X$ whose radius is $\delta$ (the last equation follows from Stokes' theorem). Since $\beta^{\prime}$ tends to zero near $\partial X$, this integral vanishes. Consequently, the cohomology class of $\beta$ is zero by Serre duality.

I want to finish this section by showing that the Nakano inequality [8] still holds for $E$-values harmonic $(0, q)$-forms on $X$. To explain this, one needs some more notation. The Lefschetz operator $L$ (which is wedging with the fundamental $(1,1)$-form for the metric $\left.h_{X}\right)$ preserves $L^{2}$-forms since the fundamental form has Poincaré growth near $\partial X$. Moreover, since $L$ is real, one has

$$
h_{x}(L \alpha, \beta) d V=h_{E}(L \alpha, \beta)=L \alpha \wedge \overline{* \beta}=\alpha \wedge \overline{\left.*^{*-1} L * \beta\right)}
$$

and so $\Lambda=*^{-1} L *$ is the formal adjoint of $L$. Since $*$ is an isometry, one concludes that also $\Lambda$ preserves the $L^{2}$-forms.

Lemma 2.5 (Nakano inequality [8]). Let $\omega \in \mathrm{H}_{(2)}^{0, k}(X, E)$. With $F_{h}$ the curvature of the metric connection on $(E, h)$ and $\Lambda$ the formal adjoint of the Lefschetz operator, one has the inequality

$$
\mathrm{i}\left\langle\Lambda F_{h} \omega, \omega\right\rangle \geq 0 .
$$

Proof. For simplicity, write $\nabla^{1,0}=\partial_{E}$ with adjoint $\partial_{E}^{*}$. One has the Kähler identity (see, e.g., $[4, \S 13]$ )

$$
\Lambda \bar{\partial}-\bar{\partial} \Lambda=-\mathrm{i} \partial_{E}^{*}
$$

which is derived in the $L^{2}$-setting as in the classical setting. Using this relation, the fact that $\omega$ is closed and co-closed $\left(\bar{\partial} \omega=0=\bar{\partial}^{*} \omega\right)$, as well as the relation

$$
F_{h}(\omega)=\bar{\partial} \partial_{E}(\omega)+\partial_{E} \bar{\partial}(\omega)=\bar{\partial} \partial_{E}(\omega),
$$

one calculates

$$
\begin{aligned}
0 \leq\left\langle\partial_{E} \omega, \partial_{E} \omega\right\rangle & =\left\langle\partial_{E}^{*} \partial_{E} \omega, \omega\right\rangle \\
& =\mathrm{i}\left\langle\Lambda \bar{\partial} \partial_{E} \omega-\bar{\partial} \Lambda \partial_{E} \omega, \omega\right\rangle \\
& =\mathrm{i}\left\langle\Lambda F_{h} \omega, \omega\right\rangle-\mathrm{i}\left\langle\Lambda \partial_{E}, \bar{\partial}^{*} \omega\right\rangle \\
& =\mathrm{i}\left\langle\Lambda F_{h} \omega, \omega\right\rangle .
\end{aligned}
$$

\section{The Calabi-Vesentini method in the $L^{2}$-Setting}

In this section I shall indicate how the method employed in $[3, \S 7, \S 8]$ to show vanishing of the groups $H^{q}\left(T_{X}\right)$ for $X$ compact can be adapted step by step in the noncompact setting.

Let $(X, h)$ be a Kähler manifold and let $T_{X}$ be the holomorphic tangent bundle. Suppose that Assumption 2.1 holds. The metric $h$ induces hermitian metrics on the bundles $\mathcal{A}_{X}^{p, q}=\wedge^{p} T_{X}^{*} \otimes \wedge^{q} \bar{T}_{X}^{*}$ of forms on $X$ of type $(p, q)$. 
The Chern connection on $T_{X}$ is the standard Levi-Civita connection and its curvature is a global $T_{X}$-valued $(1,1)$-form:

$$
F_{h} \in A_{X}^{1,1}\left(\operatorname{End}\left(T_{X}\right)\right) .
$$

Using the metric, one has an identification $\bar{T}_{X}^{*} \simeq T_{X}$ and hence $F_{h}$ induces an endomorphism of $T_{X} \otimes T_{X}$ :

$$
F_{h} \in T_{X}^{*} \otimes \bar{T}_{X}^{*} \otimes T_{X}^{*} \otimes T_{X} \simeq T_{X}^{*} \otimes T_{X}^{*} \otimes T_{X} \otimes T_{X} \simeq \operatorname{End}\left(T_{X} \otimes T_{X}\right) .
$$

One can show, using the Bianchi identity, that the resulting endomorphism vanishes on skew-symmetric tensors and hence induces

$$
Q: S^{2} T_{X} \rightarrow S^{2} T_{X}, \quad R=2 \operatorname{Tr}(Q),
$$

where the function $R$ is the scalar curvature of the metric. The operator $Q$ is selfadjoint and hence at each $x \in X$ it has real eigenvalues. Suppose that

$$
-\infty<\lambda:=\int_{x \in X} \lambda_{x}<0, \quad \lambda_{x} \text { smallest eigenvalue of } Q_{x} .
$$

The operator $Q$ together with the metric $h$ induces a Hermitian form $h_{Q}$ on the bundles $\mathcal{A}^{0, q}\left(T_{X}\right), q>0$, as follows:

$$
\begin{gathered}
h_{Q}:\left(\wedge^{q} \bar{T}_{X}^{*} \otimes T_{X}\right) \otimes\left(\wedge^{q} \bar{T}_{X}^{*} \otimes T_{X}\right) \simeq T_{X} \otimes T_{X} \otimes\left(\wedge^{q} \bar{T}_{X}^{*} \otimes \wedge^{q} \bar{T}_{X}^{*}\right) \\
\stackrel{Q}{\longrightarrow} T_{X} \otimes T_{X} \otimes\left(\wedge^{q} \bar{T}_{X}^{*} \otimes \wedge^{q} \bar{T}_{X}^{*}\right) \rightarrow \mathbf{C},
\end{gathered}
$$

where the last map is induced from the hermitian metric $h$. If $h$ is KählerEinstein, one has (see $[3, \S 8]$ )

$$
\mathrm{i} h_{x}(\Lambda F \omega, \omega)=\frac{R}{2 n}\|\omega\|^{2}-h_{Q}(\omega, \omega) .
$$

On the other hand, by [3, Lem. 3] one has the inequality

$$
h_{Q}(\omega, \omega) \geq \frac{1}{2}(q+1) \lambda_{x}\|\omega\|^{2} .
$$

In [3] it is shown that first of all $R<0$ implies $\lambda<0$, and hence, combining (6) and (5) that

$$
\mathrm{i} h_{x}(\Lambda F \omega, \omega) \leq\left(\frac{R}{2 n}-\frac{1}{2}(q+1) \lambda_{x}\right)\|\omega\|^{2} .
$$

The above function is $\leq 0$ whenever $\frac{R}{2 n}-\frac{1}{2}(q+1) \lambda<0$ and it is identically zero if and only if $\omega=0$. Now contrast this with Nakano's lemma in the form of Lemma 2.5, which holds under the assumptions of Section 2. The conclusion is:

Proposition 3.1. Suppose that Assumption 2.1 holds for a quasi projective Kähler-Einstein manifold $(X, h)$ and its holomorphic tangent bundle $\left(T_{X}, h\right)$. Suppose also that $R<0$, where $R$ is the scalar curvature. Then for all integers $q$ for which $q<\frac{R}{n \lambda}-1$, one has $\mathrm{H}_{(2)}^{0, q}\left(X, T_{X}\right)=0$. 
Remark 3.2. The above proof has to be modified slightly for $q=0$. In that case the term $h_{Q}(\omega, \omega)$ in (5) vanishes, and since $R<0$, the above argument directly shows that $\mathrm{H}_{(2)}^{0}\left(X, T_{X}\right)=0$. This implies that $\bar{X}$ admits no vectorfields tangent to $\partial X$.

\section{Application to locally symmetric VARIEties of HeRmitian type}

Let $G$ be a reductive $\mathbf{Q}$-algebraic group of hermitian type, i.e. for $K \subset G(\mathbf{R})$ maximal compact, $D=G(\mathbf{R}) / K$ is a bounded symmetric domain. Fix some neat arithmetic subgroup $\Gamma \subset G(\mathbf{Q})$ and let $X=\Gamma \backslash D$ be the corresponding locally symmetric manifold. It is quasi-projective and by [1] admits a smooth toroidal compactification $\bar{X}$ with boundary a normal crossing divisor $\partial X$.

Let $\rho: G \rightarrow \mathrm{GL}(E)$ be a finite-dimensional complex algebraic representation with $\tilde{E}_{\rho}$ the corresponding holomorphic vector bundle on $D$ and $E_{\rho}$ the bundle it defines on $X$. Fix also a $G$-equivariant hermitian metric $\tilde{h}$ on $\tilde{E}_{\rho}$ (which exists since the isotropy group of the $G(\mathbf{R})$-action on $D$ is the compact group $K$ ) and write $h$ for the induced metric on $E_{\rho}$. By [6, Thm. 3.1], there is a unique extension of $E_{\rho}$ to an algebraic vectorbundle $E_{\rho}^{\#}$ on $\bar{X}$ with the property that the metric $h$ is a so-called good metric for the bundle $E_{\rho}$ relative to $E_{\rho}^{\#}$.

For what follows it is important to observe the next lemma.

Lemma 4.1. The metric $(1,1)$-form $\omega_{h_{X}}$ of a Kähler-Einstein metric $h_{X}$ has Poincaré growth near $\partial X$.

Proof. The Kähler-Einstein condition means that

$$
\omega_{h_{X}}=-k \cdot \mathrm{i} \partial \bar{\partial} \log \left(\operatorname{det} h_{X}\right)
$$

for some positive real constant $k$. Up to some positive constant, the righthand side can be identified with the first Chern form for the canonical line bundle $\Omega_{X}^{n}$ with respect to the metric induced by $h_{X}$. Since this metric is $G(\mathbf{R})$-equivariant, it is good in Mumford's sense and so $\omega_{h_{X}}$ is also good.

Clearly, if this is to be useful in applications, given a bundle (with some $G(\mathbf{R})$-equivariant hermitian metric), one needs to get hold of the extension making the metric good. Here are some examples.

Examples 4.2. (i) Let $E=\Omega_{X}^{p}$. Then $E^{\#}=\Omega_{\frac{p}{X}}(\log \partial X)$, the bundle of $p$-forms with at most log-poles along $\partial X$. This is not trivial. See [6, Prop. 3.4] where this is shown for $p=1$. Since

$$
\Omega_{\bar{X}}^{p}(\log \partial X)=\bigwedge^{p} \Omega_{\frac{1}{X}}(\log \partial X),
$$

this implies the result for all $p$. In particular, smooth sections of $\Omega \frac{1}{X}$ are bounded near $\partial X$. Indeed, if $f=0$ is a local equation for $\partial X$ and $\omega$ a smooth section of $\Omega_{X}^{1}$, then $f \cdot \omega$ is a smooth section of $\Omega_{X}^{1}(\log \partial X)$. Then $\|f \cdot \omega\|^{2}=\|f\|^{2}\|\omega\|^{2}$ and since $\|\omega\|^{2} \leq C(\log \|f\|)^{N}$, the norm of $f \cdot \omega$ is bounded. A similar argument holds for smooth sections of $\Omega_{\frac{p}{X}}^{p}$ and hence for sections of $\mathcal{A}_{\bar{X}}^{p, q}$. 
(ii) One has $T_{X}^{\#}=T_{\bar{X}}(-\log \partial X)$, the bundle of holomorphic vector fields on $\bar{X}$ which are tangent to the boundary $\partial X$, since this is the dual of the bundle $\Omega \frac{1}{X}(\log \partial X)$. Any smooth section of this bundle is bounded near the boundary: its normal component tends to zero and the Poincaré growth of the metric implies (by compactness of $\partial X$ ) that the tangential component remains bounded.

The above examples show that the holomorphic tangent bundle $T_{X}$ satisfies Assumption 2.1 (b).

I can finally state the main result:

Theorem 4.3. Let $(\bar{X}, \partial X)$ be as before, e.g., $X=\Gamma \backslash D, D=G(\mathbf{R}) / K$ hermitian symmetric, $\Gamma$ a neat arithmetic subgroup of $G(\mathbf{Q})$ and $\bar{X}$ a good toroidal compactification with boundary $\partial D$. Let $R$ be the scalar curvature of the $G(\mathbf{R})$-equivariant (Kähler-Einstein) metric and let $\lambda$ be as before (cp. (4)). Set $\gamma(D):=R / n \lambda$. This is a positive integer and

$$
\mathrm{H}_{(2)}^{0, q}\left(X, T_{X}\right)=0 \quad \text { for all } q \text { with } q<\gamma(D)-1 .
$$

If no irreducible factor of $D$ has dimension 1 , one has $\gamma(D) \geq 3$. In particular, the resulting pairs $(\bar{X}, \partial X)$ are infinitesimally rigid.

Proof. Since $X$ admits a Kähler-Einstein metric $h_{X}$, by Lemma 4.1 its fundamental $(1,1)$-form has Poincaré growth near the boundary. So Assumption 2.1 (a) is fulfilled. By Example 4.2 (iii) the second condition is also fulfilled.

In order to apply Proposition 3.1, one observes that the Kähler manifold $X$ is homogeneous and that therefore $\lambda=\lambda_{x}, x \in X$, a constant. Since the scalar curvature of $D$ is known to be negative, this proves the result, except that one still has to show that $\gamma(D)$ is an integer $\geq 2$. The calculation of $\gamma(D)$ is local and has been done in $[2,3]$ and it indeed implies that it is an integer $\geq 2$. Also, it is shown there that $\gamma(D) \geq 3$ whenever $D$ has no irreducible factor of dimension 1. For details, see [3, §3] and [2, §2]. See also Remark 4.4 below.

I apply this to infinitesimal deformations of $(\bar{X}, \partial X)$ as follows. As is well known, these correspond bijectively to elements of $H^{1}\left(\bar{X}, T_{\bar{X}}(-\log \partial X)\right)$. See, e.g., [9, Prop. 3.4.17].

Now assume that $\alpha \in \mathcal{A}_{\bar{X}}^{0,1}\left(T_{\bar{X}}(-\log \partial X)\right)$ represents a given cohomology class $[\alpha] \in H^{1}\left(\bar{X}, T_{\bar{X}}(-\log \partial X)\right)$. By Proposition 2.4 , the class $\beta=\alpha \mid X$ is an $L^{2}$-harmonic form and it suffices to show that $\beta=0$, which follows from the vanishing of $\mathrm{H}_{(2)}^{0,1}\left(X, T_{X}\right)$.

Remark 4.4. For irreducible $D$ there is a table for the values of $\gamma(D)$ in $[3]$ and [2]. I copy their result:

\begin{tabular}{ccccccc}
\hline Type & $I_{p, q}$ & $I I_{m}, m \geq 2$ & $I I I_{m}, m \geq 1$ & $I V_{m}, m \geq 3$ & $V$ & $V I$ \\
$\gamma(\boldsymbol{D})$ & $p+q$ & $2(m-1)$ & $m+1$ & $m$ & 12 & 18 \\
$\operatorname{dim}_{\mathbf{C}} \boldsymbol{D}$ & $p q$ & $\frac{1}{2} m(m-1)$ & $\frac{1}{2} m(m+1)$ & $m$ & 16 & 27 \\
\hline
\end{tabular}


If $D=D_{1} \times \cdots \times D_{N}$ is the decomposition into irreducible factors, one has $\gamma(D)=\min _{j} \gamma\left(D_{j}\right)$. One sees from this that $\gamma(D) \geq 2$ with equality precisely when $D$ contains a factor of type $I_{1,1} \simeq I I_{2} \simeq I I I_{1}$. One also sees that the best vanishing result is for the unit ball $I_{p, 1}$ where all groups vanish.

Corollary 4.5. Under the assumptions of Theorem 4.3, the pair $(\bar{X}, \partial X)$ has a unique model over a number field.

Proof. This follows using spreads. For details see [7, 10].

Remark 4.6. The above theorem is false for Shimura curves (one-dimensional locally homogeneous algebraic manifolds). However, the corollary is true since all Shimura curves have models over $\overline{\mathbf{Q}}$. A proof which is a variant of the above method was given in [5]. This article in fact motivated this note.

Acknowledgments. Thanks to Christopher Deninger for pointing out reference [5].

\section{REFERENCES}

[1] A. Ash et al., Smooth compactifications of locally symmetric varieties, Cambridge Mathematical Library, Cambridge University Press, Cambridge, 2010. MR2590897

[2] A. Borel, On the curvature tensor of the Hermitian symmetric manifolds, Ann. of Math. (2) 71 (1960). MR0111059

[3] E. Calabi and E. Vesentini, On compact, locally symmetric Kähler manifolds, Ann. of Math. (2) 71 (1960), 472-507, MR0111058.

[4] J.-P. Demailly, $L^{2}$ Hodge theory and vanishing theorems, in Introduction à la théorie de Hodge, Panoramas et Synthèses, 3, Société Mathématique de France, Paris, 1996. MR1409818.

[5] G. Faltings, Arithmetic varieties and rigidity, in Seminar on number theory (Paris, 1982-83), 63-77, Progr. Math., 51, Birkhäuser, Boston, MA, 1984. MR0791585

[6] D. Mumford, Hirzebruch's proportionality theorem in the non-compact case, Invent. Math. 42 (1977), 239-272. MR0471627

[7] C. Peters, Rigidity of spreadings and fields of definition, preprint (2016).

[8] S. Nakano, On complex analytic vector bundles, J. Math. Soc. Japan 7 (1955), 1-12. MR0073263

[9] E. Sernesi, Deformations of algebraic schemes, Grundlehren Math. Wiss., 334, Springer, Berlin, 2006. MR2247603

[10] G. Shimura, Algebraic varieties without deformation and the Chow variety, J. Math. Soc. Japan 20 (1968), 336-341. MR0223368

Received November 11, 2016; accepted January 23, 2017

Chris Peters

Institut Fourier,

Université Grenoble Alpes,

100 rue des maths,

38610 Gières, France

E-mail: c.a.m.peters@tue.nl

Section Discrete Mathematics,

Technical University Eindhoven,

PB 513, 5600 MB Eindhoven, Netherlands 